\title{
Neurinoma del acústico (schwanoma vestibular): Revisión y actualización de la literatura
}

\author{
Acoustic neuroma (vestibular schwannoma): A literature review
}

\author{
Carlos Stott $C^{1}$, Nicolás Albertz A², Cristián Aedo B .
}

\section{RESUMEN}

日 neurinoma del acústico corresponde al tumor más frecuente del ángulo pontocerebeloso. En su etiopatogenia se involucran factores genéticos que permiten su aparición, estando en algunos casos ligados a enfermedades como la neurofibromatosis tipo 2 expresándose de manera bilateral.

Cínicamente se divide en 4 estadios que desencadenan una diversa gama sintomatológica de acuerdo al grado de compresión y ocupación del conducto auditivo interno y tronco encefálico, sin embargo la hipoacusia sigue siendo el síntoma más importante en su presentación. Estos son el estadio intracanalicular, cisternal, de compresión de tronco encefálico e hidrocefálico.

Habitualmente alcanzan un tamaño que fluctúa entre los pocos milímetros hasta más de 3 centímetros de diámetro dividiéndose en pequeños, medianos y grandes. Asimismo se ha dicho que poseen un lento crecimiento e historia natural incierta mostrando la mayoría de los estudios cifras de crecimiento entre los 1-3 mm/año.

Para la orientación diagnóstica la audiometría de tonos puros sigue siendo el examen más orientador en una primera etapa sin embargo el estudio imagenológico con resonancia magnética contrastada con gadolinio resultafundamental para la confirmación en laactualidad.

En lo que al manejo respecta existen 3 alternativas razonables dependiendo del caso en particular como son la conducta expectante, la microcirugía y la radioterapia estereotáxica o Gamma Knife. Cada una de ellas posee factores a favor como en contra, por lo que la decisión terapéutica final se tomará considerando la velocidad de crecimiento, el tamaño tumoral y sintomatología siendo fundamental las prioridades del paciente.

Palabras clave: Neurinoma del acústico, neurinoma vestibular, schwannoma vestibular, tumor del ángulo pontocerebeloso.

\section{ABSTRACT}

Acoustic neuroma is the most frequent ponto cerebellar angle tumor. Genetic factors are involved in its etiology, and in occasions its origins can be linked to diseases such as bilaterally expressing type 2-neurofibromatosis.

Cinically, acoustic neuromas can be classified in 4 stages, which result in diverse symptoms, according to the degree of brain stem and internal auditory canal compression or obstruction; however, hearing loss remains as the most important presenting symptom. The four stages are: intracanalicular, cisternal, brain stem compressive and hydrocephalic.

Frequently they reach between few millimeters to more than 3 centimeters in diameter, and are accordingly ranked as small, medium-sized, and large. They have been reported as having slow growth and uncertain natural history; in most studies growth rates fluctuate between $1-3 \mathrm{~mm} /$ year.

1 Médico Servicio Otorrinolaringología y Cirugía de Cabeza y Cuello, Hospital Clínico Universidad de Chile.

2 Interno de Medicina, Facultad de Medicina, Universidad de Chile. 
Pure tone audiometry is the procedure of choice for diagnosis orientation in the first place, however, gadolinium-contrasted magnetic resonance imaging is currently pivotal for diagnosis confirmation.

As for management, there are three reasonable alternatives, depending on a given case particularity. These are monitoring, microsurgery and stereotactic radiotherapy or gamma $k n i f e$. Each one of them has its pros and cons, and thus the final therapy decision will depend on the symptoms and tumor growth rate and size, priorizing the patient well-being.

Key words: Acoustic neuroma, vestibular neuroma, vestibular schwannoma, ponto cerebellar angle tumor.

\section{INTRODUCCIÓN}

El neurinoma del acústico, o mejor dicho schwannoma vestibular corresponde al tumor más frecuente del conducto auditivo interno y ángulo pontocerebelos $0^{1-3}$, llegando a cifras cercanas al $10 \%$ de todos los tumores endocraneanos y al $90 \%$ del ángulo pontocerebeloso, con una incidencia anual que va desde 7,8 a 12,4 tumores por millón de habitantes ${ }^{4}$. Fue descrito por primera vez en autopsia por Eduard Sandifort en 1777, quien reportó que el tumor estaba adherido al nervio auditivo, insinuándose en el conducto auditivo interno ${ }^{5}$. Es un tumor benigno originado a partir de las células de Schwann del VIII par craneano, tanto de sus porciones vestibular superior y mayormente inferior, siendo raro encontrarlo en su porción coclear ${ }^{6,7}$. Presenta una incidencia estimada de 1 en $100.000^{1}$, de manera más frecuente entre la cuarta y sexta década de la vida, pese a que existen casos reportados en pacientes jóvenes ${ }^{8}$.

Se suele presentar de manera esporádica, algunos casos familiares, y en otros puede presentarse de forma bilateral habitualmente en el contexto de una neurofibromatosis tipo $2^{9}$.
Aún existe controversia respecto a la conducta a seguir frente a estos pacientes dada su lenta, aunque muchas veces impredecible historia natural. Es así como en ciertos casos cuando no se realiza un diagnóstico oportuno, crecen en el interior del conducto auditivo interno extendiéndose al ángulo pontocerebeloso, llegando en casos extremos a comprimir el tronco cerebral.

Así también y dada la incierta evolución de este tumor, tampoco existe un real consenso acerca del mejor manejo de esta patología, siendo practicadas en la actualidad diferentes conductas, desde el manejo expectante con controles imagenológicos periódicos, resolución quirúrgica por distintas vías de abordaje y radioterapia en diferentes modalidades (Figura 1).

\section{ETIOPATOGENIA}

El neurinoma del acústico se origina de las células de Schwann de los nervios vestibulares superior 0 inferior en la zona de transición entre la mielina central y periférica (zona de Obersteiner-Redlich), que ocurre en la zona lateral del ángulo

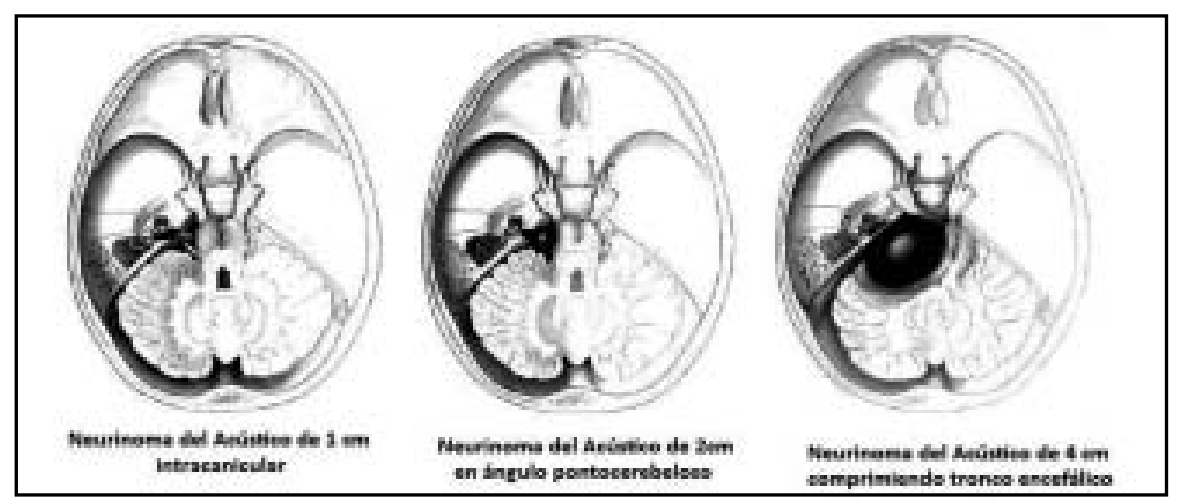

Figura 1. Diferentes presentaciones y estadio evolutivo del schwannoma vestibular. 
pontocerebeloso y medial del conducto auditivo interno. Este se origina como resultado de mutaciones en un gen supresor de tumores alojado en el brazo largo del cromosoma 22 que codifica para la proteína llamada merlina, que es la encargada de controlar la proliferación de las células de Schwann, regulando su abundancia y localización. Un solo gen funcionante de la merlina es suficiente para prevenir la formación del tumor, por lo que la aparición esporádica del tumor requiere de la mutación de ambas copias del gen de la merlina. La probabilidad de aparición de dos mutaciones espontáneas e independientes en un locus, predicen la presentación del schwannoma vestibular hacia la cuarta y sexta década.

Por otro lado los casos de neurofibromatosis tipo 2, enfermedad autosómica dominante con alta penetrancia, sólo requiere la presencia de la mutación de uno de los alelos para la aparición tumoral, presentándose frecuentemente de manera bilateral.

\section{SÍNTOMAS Y SIGNOS}

Clásicamente el cuadro clínico del neurinoma del acústico ha sido dividido en 4 estadios de acuerdo al grado de compromiso del conducto auditivo interno y cisterna pontocerebelosa dando distintos síntomas y signos de acuerdo al grado de compresión, llegando en su etapa final a un estado hidrocefálico ${ }^{10}$ (Tabla 1).

En general se pueden extender en tamaño hasta $4 \mathrm{cms}$ de diámetro considerándose pequeño cuando mide menos de 1,5 cms, moderado 1,5-3 cms y grande de $3 \mathrm{cms} 0$ más ${ }^{11}$. Por otro lado y siguiendo los criterios del Comité de Audiología y Equilibrio de la Academia Americana de Otorrinolaringología y Cirugía de Cabeza y Cuello, el tamaño tumoral se puede estimar como la raíz cuadrada del producto del diámetro mayor en dirección paralela al puente petroso, por el diámetro máximo perpendicular al primero, redondeando el resultado al 0,5 cm más cercano. Sólo se considera en esta medición la porción extracanalicular del tumor ${ }^{12}$.

La pérdida auditiva es el síntoma más frecuente, llegando a estar en el $95 \%$ de los casos, siendo en la mayoría unilateral lentamente progresiva. El patrón más frecuente corresponde a una hipoacusia sensorioneural (HSN) con pérdida de frecuencias agudas, aunque cualquier patrón puede estar presente. La hipoacusia súbita se puede presentar durante su evolución hasta en el $25 \%$ de los casos, sin embargo el schwannoma vestibular es causa de solo el $1 \%$ a $3 \%$ de éstas ${ }^{13,14}$. Otra característica es la disminución marcada de la discriminación de la palabra, destacando sin embargo que el nivel de la hipoacusia no es predictor del tamaño tumoral ${ }^{15}$. Es así como la discapacidad auditiva en pacientes con neurinoma del acústico se debe principalmente a la compresión de los vasos de la cóclea y/o del nervio coclear ${ }^{16}$. Pese a esto la audición puede mantenerse inalterada ${ }^{17}$. La clasificación de la Academia Americana incluye la pérdida auditiva en cuatro grupos según el umbral de promedio tonal puro y discriminación de la palabra como muestra la Tabla 2.

Tabla 1. Estadios clínicos del schwannoma vestibular

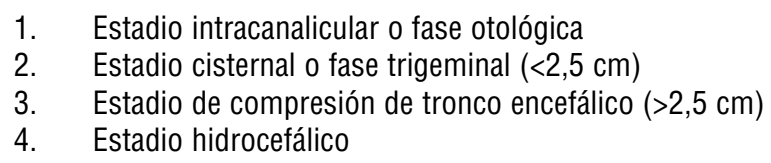

Tabla 2. Clasificación auditiva según la AAOHNS del oído enfermo

\begin{tabular}{|lcc|}
\hline Tipo & Umbrales auditivos & Discriminación verbal $(\%)$ \\
\hline$A$ & $\leq 30 \mathrm{~dB}$ & $\geq 70$ \\
B & $>30 \mathrm{~dB}, \leq 50 \mathrm{~dB}$ & $\geq 50$ \\
$\mathrm{C}$ & $>50 \mathrm{~dB}$ & $\geq 50$ \\
D & Cualquier nivel & $<50$ \\
\hline
\end{tabular}


El tinnitus es el segundo síntoma en frecuencia, presente en cerca del $65 \%$ de los pacientes, habitualmente de tono agudo y en el lado afectado por el tumor.

Por otro lado el vértigo sólo se ve en cerca del $20 \%$ en los que esta afectado el vestibular inferior, siendo más común en los tumores pequeños. Es así que es mucho más frecuente la inestabilidad y el desequilibrio.

Los síntomas derivados de los nervios facial y trigémino ocurren temporalmente después de las fallas auditivas y vestibulares y en general corresponden a tumores grandes de más de $2 \mathrm{~cm}$ de diámetro. Los pacientes tienen habitualmente parestesias faciales e incluso ausencia del reflejo corneal. Espasmos o pérdida de la fuerza en los músculos faciales se ve en el $17 \%$ de los casos. La cefalea, alteraciones del estado mental, náuseas y vómitos son propios de la etapa hidrocefálica. Además se pueden ver alterados los nervios III, IV $0 \mathrm{VI}$ causando diplopia 0 bien los nervios IX y X causando disfagia, aspiración y carraspera.

\section{DIAGNÓSTICO}

La audiometría de tonos puros es la prueba más útil en la orientación diagnóstica, evidenciando una HSN asimétrica en tonos agudos en casi el $70 \%$, sin embargo sólo el $5 \%$ de los pacientes con este patrón audiométrico padecen neurinoma del acústico ${ }^{18}$.

La discriminación de la palabra se ve alterada en casi $50 \%$ de los casos, sin embargo en tumores pequeños cerca de la mitad de los pacientes permanecen con una discriminación excelente, incluso en los tumores de mayor tamaño un tercio la mantienen cercano a lo normal. La pérdida del

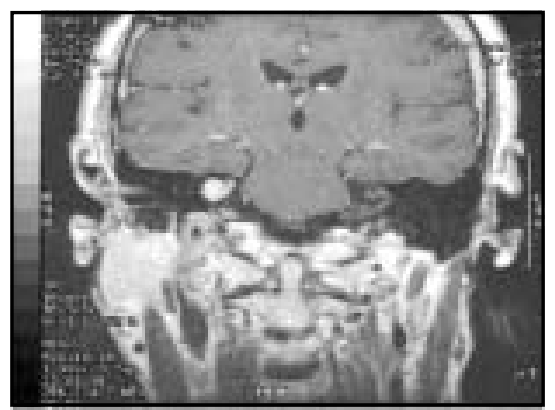

reflejo estapedial o su disminución, explorado en la impedanciometría está presente en la mayoría de los schwannomas vestibulares, pero su normalidad no excluye el diagnóstico.

También se han utilizado en el diagnóstico los potenciales evocados auditivos del tronco cerebral que en pacientes con neurinoma se aprecia ausencia o retardo de latencia en la onda $V$ en el oído afectado.

Las pruebas vestibulares en general no aportan datos significativos en el diagnóstico pudiendo encontrarse en la electronistagmografía reducción de la respuesta de la prueba calórica en el oído afectado, sin embargo carece de especificidad suficiente $^{19}$. La prueba calórica clásica, gold standard para evaluar el aparato vestibular a través del canal semicircular lateral, puede ser complementada con la prueba calórica mínima, que aporta a esta última la exploración del canal semicircular posterior, ofreciendo la posibilidad de realizar un diagnóstico anatómico según las ramas vestibulares comprometidas por el tumor ${ }^{20}$.

Pese a la orientación que brinda la audiología, la prueba con mayor impacto corresponde a la imagenología a través de la resonancia magnética (RM) con gadolinio, constituyendo actualmente el gold standard en el diagnóstico, permitiendo además la planificación prequirúrgica. Es así como característicamente en el neurinoma del acústico se observa una imagen hipointensa en $\mathrm{T} 1$ con aumento de la intensidad al agregar gadolinio. Otra alternativa es la RM con la técnica de Fast Spin Eco, obteniendo imágenes ponderadas en T2 sin la necesidad de uso de gadolinio como medio de contraste, con el consecuente menor costo, sin embargo con algo menor de sensibilidad en la detección de tumores de menor tamaño $0^{21,22}$.

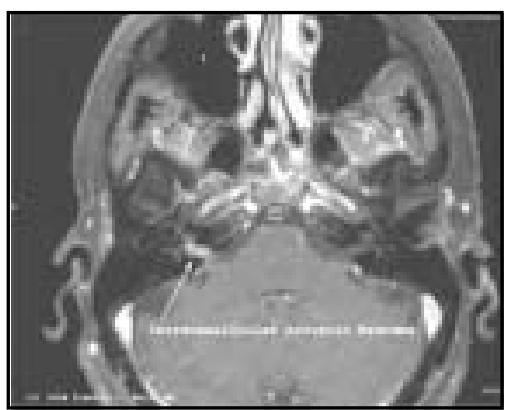

Figura 2. Resonancia magnética cerebral que evidencia la presencia de un neurinoma del acústico. (Adaptado de HAIN TC. Acoustic Neuroma. dizziness-and-hearing.com el 15 Enero 2008. http://www.dizziness-and-hearing.com)

a. RM cerebral corte coronal que muestra un neurinoma del acústico derecho. b. RM cerebral axial evidenciando un neurinoma del acústico intracanalicular derecho. 
Tabla 3. Diagnóstico diferencial del schwanoma vestibular. (Adaptado de ANIL K. LALWANI "CURRENT Diagnosis \& Treatment Otolaryngology Head and Neck Surgery", segunda edición 2007)

\begin{tabular}{ll}
\hline \multicolumn{2}{l}{ Lesiones comunes del ángulo pontocerebeloso } \\
- & Schwanomas del V, VII, VIII \\
- & Meningiomas \\
- & Epidermoides \\
Lesiones de restos congénitos \\
$-\quad$ & Epidermoides \\
- & Quistes aracnoidales \\
- & Lipomas \\
Lesiones vasculares \\
- & Hemangiomas \\
- & Paragangliomas (glomus jugulare) \\
- & Aneurismas \\
- & Hemangioblastoma \\
Tumores intracerebrales \\
- & Meduloblastomas \\
- & Astrocitomas \\
- & Gliomas \\
- & Tu del IV ventrículo \\
- & Hemangioblastomas \\
Lesiones que se extienden de la base de cráneo \\
- & Granuloma de colesterol \\
- & Glomus \\
- & Cordomas \\
- & Condrosarcomas \\
Otras alteraciones malignas \\
- & Metástasis
\end{tabular}

\section{HISTORIA NATURAL}

La caracterización de la historia natural del schwannoma vestibular ha sido motivo de controversia entre expertos dado por los diferentes criterios de medición pero sobre todo por las variaciones individuales de cada caso. Pese a esto se considera que posee una lenta velocidad de crecimiento pasando por períodos de crecimiento intercalados con otros de quiescencia e incluso disminución de tamaño.

Asimismo se han descrito distintos patrones de crecimiento. Charabi y cols. realizaron un seguimiento de 127 pacientes mostrando que $82 \%$ de los casos hubo un aumento de su volumen comparado con el medido al diagnóstico, $12 \%$ no creció manteniéndose en su tamaño original y en el $6 \%$ disminuyó de tamaño².

Por otro lado la velocidad de crecimiento, medida habitualmente en mm/año también ha tenido una importante dispersión, así por ejemplo Rosenberg describió un rango entre los $0,35 \mathrm{~mm} /$ año en sus pacientes posoperados con resección subtotal, a 0,91 mm/año en los pacientes no quirúrgicos ${ }^{23}$. En contraste encontramos grupos de pacientes con tasas de crecimiento de hasta más de $10 \mathrm{~mm} / \mathrm{añ}^{2}$.

Pese a esto la mayoría de los estudios arrojan cifras entre los 1-3 mm/año, teniendo una velocidad mayor mientras más jóvenes sean los pacientes estudiados ${ }^{8,24-27}$.

Es precisamente la velocidad de crecimiento, el tamaño tumoral y sintomatología los que determinan su manejo $0^{28}$.

\section{TRATAMIENTO}

En general en la actualidad existen 3 distintas alternativas de enfrentamiento terapéutico, sin embargo finalmente la decisión se basa en las características clínicas personales. Las opciones son básicamente manejo conservador con controles imagenológicos periódicos, cirugía para remover el tumor definitivamente y radioterapia estereotáxica o con Gamma Knife.

No hay evidencias científicas probadas sobre cuál opción es la mejor. Todos ellos son seguros y efectivos pero la decisión debe estar basada en las prioridades individuales de cada paciente ${ }^{29}$.

El manejo conservador consiste en el monitoreo clínico e imagenológico periódico y uso de audífonos en los casos que estos sean beneficiosos. Este es un método particularmente apropiado para pacientes añosos, con patologías concomitantes o con una velocidad de crecimiento anual baja que no incida en mayor sintomatología y que harían de la cirugía un mayor riesgo que beneficio ${ }^{27,30}$. Una vez diagnosticado el tumor se debe repetir el control con RM a los 6 meses y luego en intervalos anuales en caso de ser asintomáticos 0 que no presenten complicaciones $^{23,31}$.

Sin embargo con esta modalidad existe el riesgo de que se pierda la audición útil pese a que 
no exista crecimiento tumoral, eliminando así la posibilidad de ser candidato a una eventual cirugía preservadora de la audición futura.

En una segunda opción de manejo nos encontramos con la cirugía que actualmente sigue siendo la elección más deseada para erradicar la enfermedad, presentando considerables mejorías en los últimos años gracias a las técnicas de microcirugía ${ }^{32,33}$. Dentro de esta opción resulta fundamental la vía de abordaje quirúrgico que se decida realizar existiendo clásicamente tres: translaberíntica, retrosigmoidal y por fosa media realizándose una u otra dependiendo del tamaño tumoral y pretensión de preservación auditiva (Figura 3).

La vía translaberíntica implica una pérdida auditiva inevitable pero posee la ventaja de poder remover tumores de todos los tamaños con mínima retracción cerebral, además de poder visualizar y preservar el nervio facial.

Por la vía retrosigmoidal se puede obtener preservación auditiva con buena ventana quirúrgica para el ángulo pontocerebeloso, sin embargo es riesgosa por la retracción cerebral y cerebelar que implica, la imposibilidad de acceder directamente al nervio facial y las persistentes y severas cefaleas posquirúrgicas.

Por otro lado, vía fosa media se puede acceder directamente al conducto auditivo interno removiendo tumores intracanaliculares preservando la audición. Sin embargo presenta la limitación de no poder acceder a tumores cisternales de más de 1,5 cms, y al igual que la retrosigmoidal impide el correcto manejo del nervio facial, así como presenta los riesgos propios derivados de la retracción del lóbulo temporal.

De esta forma la microcirugía debe desarrollarse cuando el cirujano este seguro de la extirpación, con una relación riesgo beneficio alta.

Finalmente y como tercera opción nos encontramos con la radioterapia estereotáxica que tiene como objetivo prevenir el mayor crecimiento tumoral mientras se preservan la función auditiva y facial $^{34}$.

De igual manera la técnica de Gamma Knife puede usarse para tratar tumores residuales como también tumores en pacientes con deterioro clínico que objete la cirugía, siendo actualmente una de las mejores opciones terapéuticas, sin embargo poco accesibles en nuestro medio ${ }^{36}$.

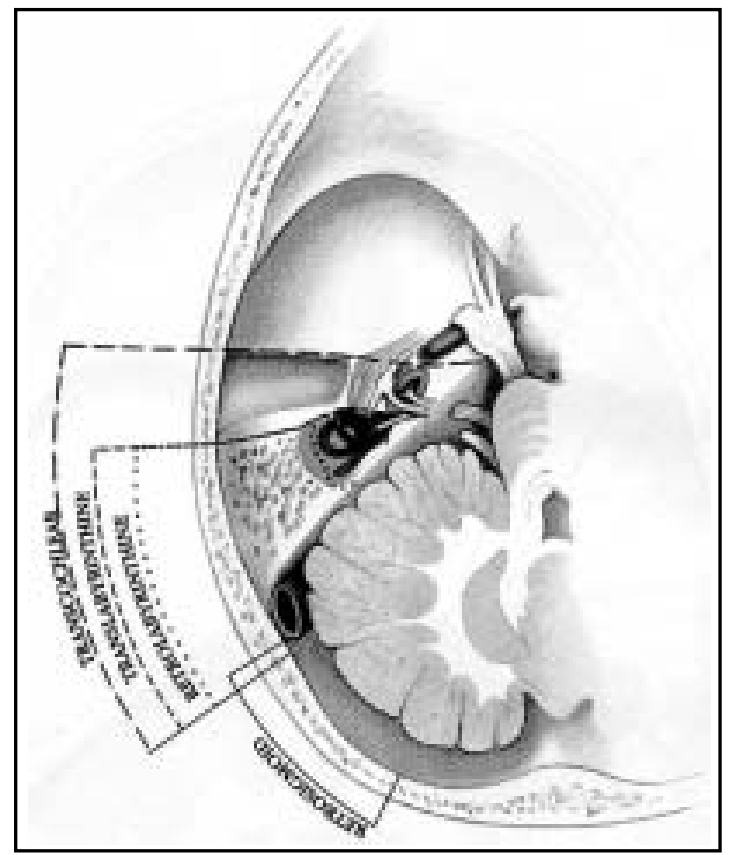

Figura 3. Vías de abordaje quirúrgicas (Extraído de ANIL K. LALWANI "CURRENT Diagnosis \& Treatment Otolaryngology Head and Neck Surgery", segunda edición 2007). 


\section{BIBLIOGRAFÍA}

1. Dazert $S$, Aletsee $C$, Brors $D$, Mlynski R, Sudhoff $H$, Hildmann H, Helms J. Rare tumors of the internal auditory canal. Eur Arch Aorhinolaryngol 2005; 262: 550-4.

2. Charabi S, Thomsen J, Tos M, Charabi B, Mantoni M, Borgesen SE. Acoustic Neuroma: Vestibular Schwannoma Growth: Past, Present and Future. Acta Otolaryngol (Stockh) 1998; 118: 327-32.

3. CHen DA. Acoustic neuroma in a private neurotology practice: trends in demographics and practice patterns. Laryngoscope 2007; 117(11): 2003-12.

4. Tos M, Charabi S, Thomsen J. Incidence of Vestibular Schwannomas. Laryngoscope 1999; 109(5): 736-40.

5. SAndifort E. Observations Anatomicao-Pathologicae. Lugduni Batavorum, 1777; Chap.IX, 116-20.

6. Komatsuzaki A, Tsunoda A. Nerve origin of the acoustic neuroma. J Laryngol Otol 2001; 115(5): 376-9.

7. Khrais T, Romano G, Sanna MJ. Nerve origin of vestibular schwannoma: a prospective study. $J$ Laryngol Otol 2007; 122: 128-31.

8. Mirzayan MJ, Gerganov VM, Lüdemann W, O S SAmII M, SAmI A. Management of vestibular schwannomas in young patients-comparison of clinical features and outcome with adult patients. Childs Nerv Syst 2007; 23: 891-5.

9. Halum SL, ERbe CB, Friedland DR, Wackym PA. Gene discovery using a human vestibular schwannoma cDNA library constructed from a patient with neurofibromatosis type 2 (NF2). Otolaryngol Head Neck Surg 2003; 128(3): 364-71.

10. Selesnick SH, JaCKLeR RK. Clinics manifestations and audiologic diagnosis of acoustic neuromas. Otolaryngol Cin North Am 1992; 25: 521-50.

11. Haln TC. Acoustic Neuroma. dizziness-andhearing.com 2008. Citado 15 Enero 2008. http://www.dizziness-and-hearing.com.

12. Committee on Hearing and Equilibrium. Committee on Hearing and Equilibrium guidelines for the evaluation of hearing preservation in acoustic neuroma (vestibular schwannoma). Otolaryngol Head Neck Surg 1995; 113: 179-80.
13. Sauvaget E, Kicl S, Kania R, Herman P, Tran BA, Huy P. Sudden sensorineural Hearing loss as a revealing symptom of vestibular Schwannoma. Acta Otolaryngol 2005; 125: 592-5.

14. Inoue Y, Kanzaki J, Ogawa K. Vestibular schwannoma presenting as sudden deafness. $J$ Laryngol Otol 2000; 114: 589-92.

15. Del Río L, Lassaletta L, Alfonso C, Sarriá MJ, GaVILÁn J. Disociación clínica-tamaño tumoral en el neurinoma del acústico: ¿realidad o problema de medida? Acta Otorrinolaringol Esp 2006; 57: 345-49.

16. Forton G, Cremers C, Offeciers E. Acoustic Neuroma Ingrowth In The Cochlerar Nerve: Does It Influence The Clinical Presentation? Ann Atol Rhinol Laryngol 2004; 113: 582-86.

17. Magdiarz D, Wiet R, Dinces E, Adamiec L. Normal audiologic presentations in patients with acoustic neuromas: An evaluation using audiologic parameters. Otolaryngol Head Neck Surg 2000; 122: 157-62.

18. Daniels RL, Swallow C, Shelton C, Davidson HC, Kresci CS, Harnsberger HR. Causes of unilateral sensorineural hearing loss screened by highresolution fast spin echo magnetic resonance imaging: review of 1070 consecutive cases. $A m$ J Otol 2000; 21: 173-80.

19. Hernández E, Fraile R, De Miguel F, Sampériz LM, EIRAS J, ORTIZ A. The role of video-nystagmography in the diagnosis of acoustic neuroma. Acta Corrinolaringol Esp. 2003; 54(6): 413-6.

20. Riveros H, Cohen M, Badia P, Anabalon Jl, Correa C. Utilidad de la prueba calórica mínima. Rev Otorrinolaringol Cir Cabeza Quello 2005; 65: 193-6.

21. Morales C. Sospecha Clínica de Neurinoma del Acústico y Correlato Imagenológico. Rev Otorrinolaringol Or Cabeza Quello 2002; 62: 6-12.

22. Plaza G, López la, Fuente J, Aparicio JM et al. Resonancia Magnética: Prueba de Elección en el Despistaje de Tumores del Conducto Auditivo Interno y Angulo Pontocerebeloso. Acta Aorrinolaringol Esp 2001; 52: 651-6.

23. Rosenberg S. Natural history of acoustic neuromas. Laryngoscope 2000; 110: 497-508.

24. Yosнiмото Y. Systematic review of the natural history of vestibular schwannoma. J Neurosurg 2005; 103(1): 59-63.

25. SaKamoto T, FukUda S, InUYAma Y. Hearing loss and 
growth rate of acoustic neuromas in follow-up observation policy. Auris Nasus Larynx 2001; 28 : 23-7.

26. Roehm PC, Gantz BJ. Management of Acoustic Neuromas in Patients 65 Years or Older Otology \& Neurotology 2007; 28: 708-14.

27. Shin YJ, Fraysee B, Cognard C et al. Effectiveness of conservative management of acoustic neuromas. AM J Ool 2000; 21: 857-62.

28. Møller P, Myrseth e, Pedersen PH, Larsen Jl, Krakenes J, Moen G. Acoustic neuromatreatment modalities. Acta Otolaryngol 2000; Suppl 543: 34-7.

29. Rutherford SA, King AT. Vestibular schwannoma management: what is the "best" option? $\mathrm{Br} \mathrm{J}$ Neurosurg 2005; 19(4): 309-16.

30. Hoistad DL, Melnik G, Mamikoglu B, Battista R, O'Connor CA, Wiet RJ. Update on conservative management of acoustic neuroma. Otology \& Neurotology 2001; 22: 682-5.
31. Perry BP, Gantz BJ, Rubinstein JT. Acoustic neuromas in the elderly. Otology \& Neurotology 2001; 22: 389-91.

32. Bennett M, HAYNes DS. Surgical approaches and complications in the removal of vestibular schwannomas. Otolaryngol Cin North Am 2007; 40(3): 589-609.

33. Wiet R, Mamikoglu B, Odom L, Hoistad D. Longterm results of the first 500 cases of acoustic neuroma surgery. Otolaryngol Head Neck Surg 2001; 124: 645-51.

34. Weil RS, Cohen JM, Portarena I, Brada M. Optimal dose of stereotactic radiosurgery for acoustic neuromas: a systematic review. $\mathrm{Br} J$ Neurosurg 2006; 20(4): 195-202.

35. Chung WY, Liu KD, Shiau CY et Al. Gamma knife surgery for vestibular schwannoma: 10-year experience of 195 cases. J Neurosurg 2005; 102 Suppl: 87-96. 